\title{
SHELL-MODEL COULOMB ENERGIES WITH DEFORMATION
}

\author{
J. JÄNECKE and E. COMAY \\ Department of Physics, The University of Michigan, Ann Arbor, MI 48109, USA
}

Received 28 November 1983

\begin{abstract}
A shell-model Coulomb energy equation which includes deformation effects has been obtained. It describes over 250 experimental Coulomb displacement energies with $4<A<240$ with a standard deviation of $\sigma=41 \mathrm{keV}$ ( $\sigma=33 \mathrm{keV}$ for $Z>20$ ). The Coulomb interactions between valence-core and valence-valence protons are used as adjustable parameters while deformation parameters are derived from other experimental sources.
\end{abstract}

Expressions for the total Coulomb energies and Coulomb displacement energies have been derived recently [1]. They apply also to the iso-scalar, isovector and iso-tensor Coulomb energies and to nuclei with protons and neutrons in the same and in different major shell regions. Special attention was given to (i) the problem of properly including the Coulomb interactions between core-core, corevalence and valence-valence protons and (ii) the problem of properly accounting for the change in nuclear size which affects the strength of the interaction energies. Systematic deviations were observed in the regions near $A \approx 100$ and $A \approx 160$. It is the purpose of the present work to interpret and describe these effects as the result of deformed nuclear shapes.

For diagonal regions $(i, i)$ where protons and neutrons occupy orbits in the same major shells, Coulomb displacement energies are written as [2]

$$
\begin{aligned}
& \Delta E_{\mathrm{c}}(Z, N)=S(Z, N)\left\{\omega_{\overline{i-1} i} Z_{i}+\omega_{i i}\left(Z-Z_{i}\right)\right. \\
& \left.\quad+\pi_{i}\left[\frac{1}{2} \delta_{\mathrm{oe}} / T+\frac{1}{2} \delta_{\mathrm{oo}}\right]\right\} .
\end{aligned}
$$

For off-diagonal regions $(i, i+1)$ they are written as [1]

$$
\begin{aligned}
& \Delta E_{\mathrm{c}}\left(Z_{i}-H, N_{i}+P\right)=S\left(Z_{i}-H, N_{i}+P\right) \\
& \quad \times\left(\frac{H}{P+H}\left[\omega_{i-1} Z_{i}+\omega_{i i}\left(Z-Z_{i}\right)+H^{-1} \pi_{i} \delta_{H}\right]\right. \\
& \left.\quad+\frac{P}{P+H} \omega_{\bar{i} i+1} Z\right) .
\end{aligned}
$$

Here, $S(Z, N)$ is a common factor, and $\Omega_{\bar{i}-1} \overline{i-1}(Z, N)$ $\equiv S(Z, N) \omega_{\overrightarrow{i-1}} \overrightarrow{i-1}, \Omega \overline{i-1} i(Z, N) \equiv S(Z, N) \omega_{\overline{i-1} i}$ and $\Omega_{i i}(Z, N) \equiv S(Z, N) \omega_{i i}$ are the size-corrected average core-core, core-valence, and valence-valence Coulomb interaction energies, and $\Pi_{i}(Z, N)=$ $S(Z, N) \pi_{i}$ is the size-corrected pairing interaction energy of $(J=0)$-coupled valence protons. $\left(\Omega_{\bar{i}-1} \overline{i-1}\right.$ is used only for $E_{\mathrm{c}}(Z, N)$.) Furthermore, $P$ and $H$ are the number of particles and holes in off-diagonal regions. The quantities $\delta_{\mathrm{oe}}, \delta_{\mathrm{oo}}$ and $\delta_{H}$ are unity for odd- $Z$ /even- $N$, odd/odd and even- $H$ nuclei, respectively, and zero otherwise. The magic numbers are $Z_{2}=N_{2}=2, Z_{3}=N_{3}=8$, and so on.

Details of eqs. (1) and (2) including expressions for the total Coulomb energies and the iso-scalar, iso-vector, and iso-tensor energies are discussed else. where [1]. It is also pointed out that the interaction energies $\Omega$ contain systematic errors of 3 to $10 \%$, particularly for light nuclei, because other small effects $[3,4]$, such as charge-dependent nuclear forces, are not properly represented by eqs. (1) and (2).

The size factor $S(Z, N)$ plays an important role in the application of the equations. It was observed that a simple decrease proportional to $A^{-\sigma / 3}$ with $\sigma$ usually in the range from zero to one gives good agreement in diagonal regions,

$$
S(Z, N)=S\left(Z_{i}, N_{i}\right)\left[\left(Z_{i}+N_{i}\right) /(Z+N)\right]^{\sigma_{i} / 3} .
$$

Characteristics of several expressions were explored for off-diagonal regions, and it was found that size 
factors which preserve the isotope and isotone shifts of the neighboring diagonal regions lead to the most satisfactory results,

$$
\begin{aligned}
& S(Z, N)=S\left(Z_{i}, N_{i}\right)\left[\left(Z_{i}+N_{i}\right) /\left(Z+N_{i+1}\right)\right]^{\sigma_{i / 3}} . \\
& \quad \times\left[\left(Z+N_{i+1}\right) /(Z+N)\right]^{\sigma_{i+1 / 3}} .
\end{aligned}
$$

Therefore, no new parameters are introduced in the off-diagonal regions.

Two types of analyses were carried out. In procedure (A), the three (or four) parameters $\sigma_{i}, \omega_{\overline{i-1} i}$, $\omega_{i i}$ (and $\pi_{i i}$ ) were determined independently for each diagonal region, and results for the off-diagonal regions were obtained by extrapolation with eq. (2). In the global procedure (B), all 21 parameters were determined simultaneously. While good overall agreement was obtained with procedure (B) with a standard deviation of about $45 \mathrm{keV}$, procedure (A) resulted in a gradual systematic deterioration in the extrapolated regions. A few data points near ${ }^{16} \mathrm{O}$ with $Z<8, N$ $>8$ displayed deviations $\epsilon$ ranging to $-200 \mathrm{keV}$. This effect persists in the present calculations and is likely the result of structure effects which are for these light nuclei not properly included in the simple equations. More importantly, the approximately 100 data points in the regions $(5,6)$ and $(6,7)$ centered near $A \approx 100$ and $A \approx 160$, respectively, displayed systematic mean deviations of $\langle\epsilon\rangle \approx-75 \mathrm{keV}$. For all extrapolated offdiagonal regions the result was $\langle\epsilon\rangle \approx-60 \mathrm{keV}$ (see table 1). It has been suggested earlier [1] that these deviations may result from changes in nuclear shape.

The present work is intended to incorporate deformation effects into the model within the adopted framework of equations. The factor $\left(1-\beta_{2}^{2} / 4 \pi\right)$ is quite successful in describing the change in Coulomb displacement energies on account of quadrupole deformation. It is the leading term of a more general expression $[5,6]$ which includes higher-order terms and terms with $\beta_{4}$. The simplest way to include deformation effects consists of multiplying eqs. (1) and (2) by a factor

$$
D(Z, N)=1-\beta_{2}^{2}(Z, N) / 4 \pi \text {. }
$$

Table 1

Mean values $\langle\epsilon\rangle$ and standard deviations $\sigma_{\epsilon}$ for the differences $\epsilon$ between experimental and calculated Coulomb displacement

\begin{tabular}{|c|c|c|c|c|c|c|c|}
\hline \multirow[t]{2}{*}{ Region } & \multirow[t]{2}{*}{$\begin{array}{l}\text { Number } \\
\text { of cases }\end{array}$} & \multicolumn{2}{|c|}{$\begin{array}{l}\text { Procedure (A) } \\
\text { (no deformation) }\end{array}$} & \multicolumn{2}{|c|}{$\begin{array}{l}\text { Procedure (A) } \\
\text { (with deformation) }\end{array}$} & \multicolumn{2}{|c|}{$\begin{array}{l}\text { Procedure (B) } \\
\text { (with deformation) }\end{array}$} \\
\hline & & $\langle\epsilon\rangle(\mathrm{keV})$ & $\sigma_{\epsilon}(\mathrm{keV})$ & $\langle\epsilon\rangle(\mathrm{keV})$ & $\sigma_{\epsilon}(\mathrm{keV})$ & (द) $(\mathrm{keV})$ & $\sigma_{\epsilon}(\mathrm{keV})$ \\
\hline$(2,2)$ & 14 & -9.3 & 82.9 & -9.3 & 83.6 & 2.6 & 79.9 \\
\hline$(3,3)$ & 39 & 5.0 & 50.5 & 5.8 & 58.0 & 12.5 & 56.3 \\
\hline$(4,4)$ & 33 & 4.8 & 29.7 & 5.4 & 32.4 & 9.0 & 33.0 \\
\hline$(5,5)$ & 33 & -0.7 & 41.0 & -0.3 & 34.8 & 3.2 & 42.7 \\
\hline$(6,6)$ & 29 & 1.7 & 18.2 & 1.7 & 15.4 & 11.0 & 17.3 \\
\hline$(7,7)$ & $5(8)$ & 0.0 & 43.1 & 10.4 & 42.0 & -29.7 & 63.5 \\
\hline diagonal & 153 & 1.6 & 42.4 & 2.4 & 43.9 & 7.2 & 46.0 \\
\hline$(2,3)$ & 4 & -95.0 & 68.9 & -92.8 & 71.3 & -67.6 & 117.8 \\
\hline$(3,4)$ & 10 & -34.1 & 27.5 & -38.4 & 28.2 & -25.6 & 34.0 \\
\hline$(4,5)$ & 20 & 10.8 & 24.9 & 7.9 & 24.6 & 3.6 & 22.5 \\
\hline$(5,6)$ & 40 & -76.3 & 48.3 & -57.8 & 38.1 & -9.6 & 32.0 \\
\hline$(6,7)$ & 58 & -71.4 & 62.9 & -8.3 & 40.9 & 1.9 & 29.1 \\
\hline$(7,8)$ & 3 & & & $-17.4^{a)}$ & $59.2^{a)}$ & -14.5 & 34.0 \\
\hline off-diagonal & 135 & -58.3 & 60.4 & -25.5 & 46.9 & -5.7 & 33.9 \\
\hline total & 288 & -25.8 & 60.6 & -10.7 & 47.5 & 1.1 & 41.2 \\
\hline total b) & 221 & & & -12.1 & 40.4 & 0.3 & 32.7 \\
\hline
\end{tabular}
energies. In procedure (A) each diagonal region is fitted individually while the off-diagonal regions are obtained by extrapolation. Procedure (B) represents a global fit. The results without deformation are from ref. [1].

a) Not extrapolated.

b) Only for nuclei with $Z>20$. The number of parameters is reduced to 13 . 
The deformation factor $D(Z, N)$ reflects the combined influence [7] of the monopole redistribution of the charge, factor $\left(1-\frac{5}{2} \beta^{2} / 4 \pi\right)$, which is partly compen. sated by the interaction between the quadrupole parts of the electrostatic potential and the charge distribution, factor $\left(1+\frac{3}{2} \beta^{2} / 4 \pi\right)$. For practical applications it becomes desirable to use a parametric representation of the nuclear deformation parameters $\beta_{2}$. Based on theoretical considerations of Bertsch [8], a simple expression has been derived [9] and applied to the rare-earth nuclei,

$\beta_{2}=\alpha_{1} x(1-x)(2-x) y(1-y)(2-y)+\alpha_{2}$.

Here, $x$ and $y$ are the fractional occupation numbers for protons and neutrons in a major shell region [for example $x=(Z-50) / 32, y=(N-82) / 44]$. More recently essentially all regions of nuclei were included in the analysis. The data for $\beta_{2}$ are surprisingly well reproduced by eq. (6) with adjusted parameters $\alpha_{1}$ and $\alpha_{2}$, and it was found that the global expression $\alpha_{2}=1.8 A^{-0.6}$ yields even better agreement within shell regions than individually adjusted constant values. The factors $\alpha_{1}$ display systematic behavior as well. In the diagonal regions the parameters are $\alpha(2,2)$ $=1.80, \alpha(3,3)=1.11, \alpha(4,4)=0.93, \alpha(5,5)=1.03$, $\alpha(6,6)=1.58, \alpha(7,7)=1.70$. In the off-diagonal regions the parameters are $\alpha(2,3)=1.35, \alpha(3,4)=0.61$, $\alpha(4,5)=0.89, \alpha(5,6)=1.40, \alpha(6,7)=1.72, \alpha(7,8)$ $=1.44$.

Results were obtained for procedures (A) and (B) including the deformation factor of eq. (5) and $\beta_{2}$ of eq. (6). The differences $\epsilon$ between the experimental and calculated Coulomb displacement energies of procedure (A) are plotted in fig. 1 with increments of 40 $\mathrm{keV}$. The mean values $\langle\epsilon\rangle$ and standard deviations $\sigma_{\epsilon}$ are included in table 1 . Table 1 shows that by including deformation effects the good agreement for diagonal regions remains essentially unchanged. However, a significant improvement is achieved for the extrapolated off-diagonal regions, particularly for the rare-

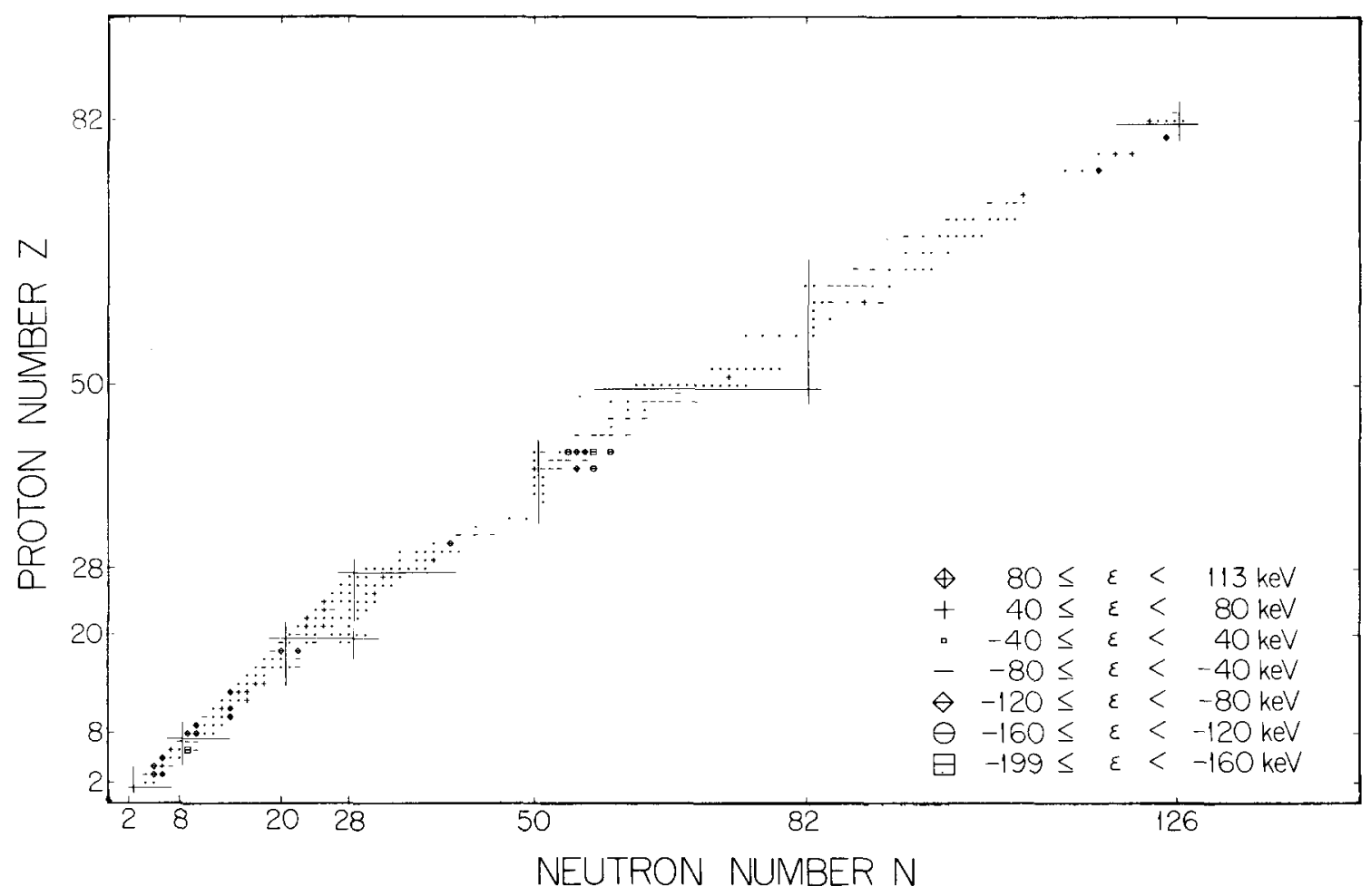

Fig. 1. Differences between experimental and calculated Coulomb displacement energies based on the shell-model equations (1) and (2) with deformation corrections. The results are for procedure (A) where the off-diagonal regions are obtained by extrapolation (see text). 
Table 2

Average matrix elements $\Omega_{i i}\left(Z_{i}, N_{i}\right)=S_{i} \omega_{i i}$ and $\Pi_{i}\left(Z_{i}, N_{i}\right)=S_{i} \pi_{i}$ for the Coulomb interactions between core-core, core-valence, and valence-valence protons in major shell regions obtained from the global analysis. The size factors $S(Z, N)$ are defined in the text and $S_{i} \equiv S\left(Z_{i}, N_{i}\right)$. The quoted digits have no physical significance but are included to facilitate numerical calculations.

\begin{tabular}{clllrr}
\hline$Z_{i}=N_{i}$ & $\sigma$ & $\Omega_{\mathrm{cc}}(\mathrm{keV})$ & $\Omega_{\mathrm{cv}}(\mathrm{keV})$ & $\Omega_{\mathrm{Vv}}(\mathrm{keV})$ & $\pi_{\mathrm{vv}}(\mathrm{keV})$ \\
\hline 2 & 0.68646 & 876.000 & 490.113 & 723.159 & 311.824 \\
8 & 1.10563 & 477.700 & 468.860 & 579.610 & 112.868 \\
20 & 0.34274 & 364.282 & 365.965 & 348.598 & 82.852 \\
28 & 0.75903 & 350.232 & 343.318 & 319.663 & 0.000 \\
50 & 0.91433 & 294.161 & 290.094 & 286.836 & 0.000 \\
82 & 1.10776 & 250.224 & 251.530 & 243.231 b) & 0.000 \\
126 a) & $(1.13804)$ a) & 213.243 a) & 214.636 a, b) & &
\end{tabular}

a) Parameters obtained from nuclei in the off-diagonal region $(7,8)$ with $Z \geqslant 82, N \geqslant 126$. The exponent $\sigma$ of the size factor is very uncertain, about \pm 0.3 .

b) Values fixed relative to preceding shell.

earth nuclei where the averaged systematic residuals are reduced from $\langle\epsilon\rangle=-71 \pm 63 \mathrm{keV}$ to $-8 \pm 41 \mathrm{keV}$. For the region near $A \approx 100$ they are reduced from $\langle\epsilon\rangle=-76 \pm 48 \mathrm{keV}$ to $-58 \pm 38 \mathrm{keV}$. Fig. 1 reflects the individual behavior. The agreement for region $(5,6)$ could be improved by increasing $\alpha_{1}(5,6)$, but this is not justified by the deformation data. By including deformation effects, the overall agreement for all extrapolated off-diagonal regions improves from $\langle\epsilon\rangle=-58 \pm 60 \mathrm{keV}$ to $-26 \pm 47 \mathrm{keV}$, thus greatly reducing the systematic error.

A further improvement is achieved with the global procedure (B). The standard deviation between all 288 experimental energies and the calculated values is $\sigma_{\epsilon}=41 \mathrm{keV}$. For the 221 data with $Z>20$ the standard deviation is $\sigma_{\epsilon}=33 \mathrm{keV}$. The interaction energies deduced from procedure (B) are listed in table 2 . The change of the parameters from procedure (A) (which uses only a subset of the data) to (B) is much smaller than observed in the earlier analysis without deformation [1]. This increases the confidence in the interaction energies from the present analysis. Only 21 quantities $\sigma, \omega_{\mathrm{cv}}, \omega_{\mathrm{vv}}$ and $\pi_{\mathrm{vv}}$ were actually used as free parameters, and only 13 for $Z>20$. The scarcity of data beyond $208 \mathrm{~Pb}$ made it necessary to adjust $\omega_{\mathrm{cv}}$ and $\omega_{\mathrm{vv}}$ of table 2 in the respective heaviest regions by introducing a reasonable fixed decrease with $A$ relative to the preceding regions.

It is interesting to note that the decrease of the interaction energies with mass number $A$ becomes increasingly stronger within each major shell region beginning in the region of $\mathrm{Ni}$ isotopes. Beyond the $\mathrm{Pb}$ isotopes the decrease is even slightly stronger than $A^{-1 / 3}$ suggesting that the charge radii may increase more rapidly than $A^{1 / 3}$ possibly due to the increased instability towards $\alpha$-decay and fission. It would be desirable to have more data in this region to substantiate this finding.

Several applications of the Coulomb energy equations such as their use in binding energy formulas have been discussed elsewhere [1].

In summary, shell-model Coulomb energy equations with deformation corrections have been used to describe the experimental Coulomb displacement energies. Using the Coulomb interaction energies between core-valence and valence-valence protons as adjustable parameters, agreement between experimental and calculated Coulomb displacement energies is achieved with a standard deviation of $41 \mathrm{keV}$ for $Z$ $>2$ and $33 \mathrm{keV}$ for $Z>20$. This appears to be better than for other known methods. The quality of extrapolations is another desirable feature of the present equations. The interaction energies from this work can also be used [1] to calculate total Coulomb energies and iso-scalar, iso-vector and iso-tensor energies.

Discussions with K.T. Hecht are appreciated. This work was supported in part by the US National Science Foundation and the United States-Israel Binational Science Foundation.

\section{References}

[1] E. Comay and J. Jänecke, Nucl. Phys. A410 (1983) 103. 
[2] K.T. Hecht, Nucl. Phys. A102 (1967) 11; A114 (1968) 280.

[3] J.A. Nolen and J.P. Schiffer, Ann. Rev. Nucl. Sci. 19 (1969) 471.

[4] N. Auerbach, J. Hüfner, A.K. Kerman and C.M. Shakin, Rev. Mod. Phys. 44 (1972) 48.

[5] R.W. Hasse, Ann. Phys. 68 (1971) 377.
[6] J. Jänecke, E.H.L. Aarts, A.G. Drentje, M.N. Harakeh and C. Gaarde, Nucl. Phys. A394 (1983) 39.

[7] L. Wilets, Theories of nuclear fission (Clarendon, Oxford, 1964) p. 18.

[8] G.F. Bertsch, Phys. Lett. 26B (1968) 130.

[9] J. Jänecke, Phys. Lett. 103B (1981) 1; and unpublished. 